\title{
Lidil
}

Revue de linguistique et de didactique des langues

43 | 2011

Le rapport au savoir dans les discours professionnels

\section{Le récit biographique en formation : un discours professionnel valorisant les parcours}

\section{Frédéric Torterat}

\section{(2) OpenEdition}

\section{Journals}

Édition électronique

URL : http://journals.openedition.org/lidil/3110

DOI : 10.4000/lidil.3110

ISSN : $1960-6052$

Éditeur

UGA Éditions/Université Grenoble Alpes

\section{Édition imprimée}

Date de publication : 30 mai 2011

Pagination : 75-88

ISBN : 978-2-84310-201-1

ISSN : $1146-6480$

\section{Référence électronique}

Frédéric Torterat, «Le récit biographique en formation : un discours professionnel valorisant les parcours », Lidil [En ligne], 43 | 2011, mis en ligne le 30 novembre 2012, consulté le 01 mai 2019. URL: http://journals.openedition.org/lidil/3110; DOI : 10.4000/lidil.3110 


\title{
Le récit biographique en formation : un discours professionnel valorisant les parcours
}

\author{
Frédéric Torterat*
}

\begin{abstract}
RÉSUMÉ
Cet article décrit les spécificités du récit biographique en formation, et analyse, à partir d'une recherche-action menée en 2007 auprès de 183 enseignants, les apports de ce type de production, plus particulièrement dans la perspective d'une formation tout au long de la vie. À ce titre, cette contribution tente de montrer dans quelle mesure les récits de vie, quand ils sont envisagés et pratiqués comme des discours professionnels, donnent un éclairage inédit sur les savoirs d'expérience et permettent de reformuler le rapport aux savoirs et aux savoir-être. Elle décrit par ailleurs les tendances qui se dégagent de l'analyse du corpus, à savoir l'autonarration, l'autocommentaire et le refus, lesquels occupent des proportions diverses et répondent, semble-t-il, à des demandes sociales différentes.
\end{abstract}

\section{ABSTRACT}

This paper describes biographical narratives produced during training sessions, on the occasion of an action-research organized in 2007 with 183 teachers. It analyses this type of production in particular in a Lifelong Training Process. In this perspective, this contribution tries to show how biographical narratives, when they are envisaged as professional discourses, conveniently enlighten knowhow through experience, and invite to modify the relationship with knowledge and professional behaviour. This paper examines moreover the different trends which appear in corpus analysis, to know self narrative, self commentary and refusal, which take diverse proportions and answer different social requests.

Le recours aux récits biographiques ne s'est vraiment répandu en formation qu'à partir des années 1990 (De Villers, 1993; Bertaux, 1997; Delory-Momberger, 2000). Cela est dû pour partie au fait que ce type

* Université de Nice, IUFM. 
de production implique les personnes d'une manière qui peut être jugée plus directe, éventuellement plus intrusive, que les récits produits en analyse de pratiques ou à la suite d'entretiens d'accompagnement (Demazière, 2008). Par ailleurs, la démarche réflexive qui s'appuie sur les récits biographiques porte non pas sur la pratique, mais sur les parcours, avec des cas de (re)conversion professionnelle qui sont diversement ressentis.

Plus spécifiquement en tant que narration sur soi, le récit de vie suppose une approche individuée du rapport au savoir et aux données d'expérience, même si, en formation, il s'inscrit généralement dans une démarche collective. Ce qui distingue ce type de support de ceux qui permettent avant tout de consigner des savoirs en lien avec des ressources documentaires et des pratiques de terrain, consiste en ce que les identités narratives qui s'y dessinent témoignent principalement de l'intégration d'un (ou de plusieurs) cadre(s) de travail, mais aussi, précisément, d'un rapport au métier. Le contexte de production du récit de vie va donc influencer plus ou moins sa «configuration», pour reprendre le terme de Ricœur (1975), en ce qu'il renvoie à un écrit tout à fait spécifique, en rapport, plus particulièrement, avec les savoir-être et les valeurs propres à telle ou telle profession.

Dans cet article, qui entend démontrer comment ce type de production contribue à valoriser les parcours personnels, nous rappellerons ce qui fonde les spécificités des récits biographiques en tant que discours professionnels. Nous exposerons ensuite, à partir d'un corpus de 183 productions recueillies en contexte de formation, les principales pratiques discursives qui s'en dégagent et ce qu'elles révèlent, par ailleurs, en termes de référence au métier et aux savoirs d'expérience ${ }^{1}$.

\section{Pratiques du récit biographique en formation : quelques rappels}

Contrairement à certains supports qui suscitent une réflexion sur le rapport aux savoirs académiques, et notamment sur les conditions de leur transmission ou de leur construction, les récits sur soi invitent leurs auteurs à ré-évènementialiser leur expérience individuelle, faisant ainsi coïncider les éléments de discours avec ceux d'un parcours qu'ils ont

1. Nous remercions Nathalie Blanc et Alain Rabatel pour leurs précieuses remarques et suggestions, sur une précédente version de cet article. 
plus ou moins de facilité à s'approprier (ou à se réapproprier). Cette exigence fait dire à Pineau (2006) que le récit biographique s'assimile à une «épreuve pragmatique performative» (p. 331), qu'il convient, selon lui, d'aborder dans une dimension dialogique. D'une manière générale, le récit de vie en formation correspond à une production personnelle à la fois biographique et diégétique, plus ou moins narrativisée, qui rend compte, intégralement ou en partie, du déroulement d'un parcours individuel de professionnalisation et de formation ${ }^{2}$. Ces caractéristiques, variablement décrites par Legrand (1993), Pineau (2002) et DeloryMomberger (2005), rendent ce type de production favorable au questionnement sur le rapport au métier, d'autant qu'elle conduit à établir des liens entre les savoirs partagés et les expériences singulières qui accompagnent les personnes tout au long de la vie.

Dans les domaines de l'enseignement et de l'éducation, la prise en compte du récit de vie comme discours professionnel est confrontée au fait que la formation concerne surtout des «questions de terrain», ce qui empêche la plupart du temps ce type de production d'intervenir dans l'évaluation de l'expérience et dans la validation des acquis (Cros, 2006; Torterat, 2008). De plus, là où d'autres types d'écrits, comme les mémoires professionnels, favorisent, chez les enseignants ou les éducateurs stagiaires en particulier, une confrontation des apports de la recherche (Blanchard-Laville et Fablet, 2000; Blanc et Varga, 2006), les récits de vie n'en font généralement pas cas, de la même manière d'ailleurs qu'ils se tiennent à priori à l'écart de toute «description minutieuse des faits présentés» (Guigue, 2000).

Outre les approches méthodologiques relevées par Bliez-Sullerot et Mevel (2004), auxquelles nous nous reportons, plusieurs auteurs ont pris position sur cette question de l'intégration des récits de vie dans les cursus de professionnalisation. Ainsi, parlant pour sa part d'«approche biographique», De Halleux (2008), qui les emploie auprès d'éducateurs spécialisés en Belgique, répertorie plusieurs «dimensions» qui, selon elle, permettent d'envisager les manières dont les récits de vie contribuent à «accroitre le professionnalisme» (p. 162) des participants. Elle recense dans ce cadre ce qu'elle désigne à travers «l'implication» (qui

2. Rappelons qu'est biographique ce qui renvoie à la vie d'un individu, et diégétique ce qui relève d'une narration marquée par des évènements singuliers. Le récit de vie est donc diégétique en ceci qu'il rapporte une histoire singulière, et biographique en ceci qu'il renvoie au vécu d'une personne. 
renvoie à l'analyse et aux démarches mises en œuvre en réponse aux questions soumises), «la relation» (qui peut répondre aux conditions d'une aide particulière ou d'un accompagnement spécifique), mais aussi d'autres variables telles que «la recherche de sens» ou «le caractère concret du réel appréhendé dans le quotidien» (p. 162-163). De Halleux évalue la productivité des récits de vie qui lui sont soumis suivant l'implication des participants dans une «démarche» qui consiste à «prendre du recul, mettre de l'ordre, faire des liens, et apporter de la cohérence dans [le] parcours» (p. 170), d'autant qu'il s'agit avant tout de faire «dialoguer vie personnelle et vie professionnelle» (p. 171).

Une telle approche dans la formation, de notre point de vue, représente une opportunité réelle pour référer à l'expérience en dépassant le caractère quelquefois préconstruit des comptes rendus et des rapports. Elle appelle aussi les participants, non pas à dégager les éléments récurrents d'une pratique ou d'un vécu quotidien, mais à se positionner dans un parcours, et à décrire, à partir d'un ensemble d'évènements singuliers, un «fil conducteur» (Lindón, 2005) qui les conduit à porter un regard réflexif sur leur identité professionnelle. Nous faisons ici l'hypothèse que la production de récits biographiques permet d'autre part de reformuler, pour ainsi dire de resignifier le rapport aux savoirs expérientiels, en marge du fait que cette démarche demande du temps en formation, comme en témoignent les éléments de corpus que nous soumettons ci-après. Ces derniers, effectivement, montrent qu'il s'y dégage plusieurs pratiques discursives, dont les apports ne sont pas analogues, et que nous résumerons en trois tendances générales.

\section{Une approche spécifique de la professionnalisation}

\section{Un dialogue entre savoirs et expériences concrètes}

Quand il est employé en formation, le récit biographique invite les personnes concernées, au premier rang desquelles les enseignants et les éducateurs, à revenir sur des évènements déterminants de leur parcours individuel. Cet exercice les amène aussi à dégager les moments intermédiaires et les diverses voies empruntées pour intégrer un ensemble d'aspirations et de valeurs communes. Les évènements, identifiés ou non comme tels, sont pour ainsi dire mis en intrigue, quelquefois en dehors même de tout renvoi au temps historique, avec ce que cela suppose de reconstruction de soi. Au demeurant, rappelons que les intervenants qui sollicitent les récits de vie (et les encadrent en partie) dans ces métiers, 
s'inscrivent dans une démarche qui consiste moins à susciter une remédiation, qu'à conforter un processus d'intégration ou d'insertion.

Remarquons qu'à l'université, et plus particulièrement dans les composantes IUFM pour ce qui relève du contexte français, la semestrialisation des parcours de formation facilite à certains égards le recours aux récits de vie dans les cursus de professionnalisation ${ }^{3}$. D'après les informations dont nous disposons, les éléments de professionnalisation (analyse de pratiques, méthodologie de l'intervention, visites, etc.) s'inscrivent dans un suivi où l'évaluation reste en partie flexible. De ce fait, une place peut être laissée à des temps de réflexion sur les liens qui s'établissent entre parcours personnels et parcours professionnels. De tels moments dans le processus de construction des compétences permettent, au-delà des savoirs et des savoir faire, de réfléchir également sur ce qui constitue les savoir-être, même si ces exigences de l'exercice ne vont pas sans poser quelques difficultés de mise en place.

Dans le domaine de l'accompagnement des stages par exemple, l'invitation à soumettre ses pratiques à un «regard critique» soulève la question de savoir en quoi cette exigence consiste vraiment ${ }^{4}$. De même, les récits de pratiques partent souvent de questionnements sur des objets spécifiques, avec un regard sur des actions et des schèmes d'actions soumis à la confrontation, ce qui s'explique notamment par le fait qu'il s'agit d'apporter des réponses concrètes à des questions portant sur un vécu quotidien. Pourtant, dans la mesure où la productivité des formations est souvent conditionnée par la complémentarité entre différentes approches de la profession, le récit biographique représente la possibilité, pour les étudiants et (les) stagiaires, de se questionner précisément sur ce en quoi consiste ce regard, autrement dit de se positionner dans un parcours qui ne commence pas au moment de l'intégration d'un cadre de travail, mais qui lui préexiste en partie.

3. Les IUFM (Instituts universitaires de formation des maitres) sont, depuis 2007-2008, des écoles intégrées dans les universités françaises.

4. Dans cette vue et concernant les écrits du milieu professionnel en général, une répartition de l'approche en «récit» d'un côté, et «commentaire» de l'autre ne permet de dépasser les schémas préconstruits que si elle s'assortit d'une réécriture non seulement du récit, mais aussi du commentaire luimême. La description d'une pratique professionnelle exige en soi un recul dont on ne dispose pas forcément lors de l'entrée dans le métier, et des éléments descriptifs qui paraitront secondaires, voire incongrus ou tout simplement anodins, ne sont pas toujours présentés au premier abord dans ce qu'ils ont de significatif (Snoeckx, 2007; Torterat, 2009). 
Les productions auxquelles nous renvoyons ici, à titre d'exemplification, ont été recueillies à l'occasion d'une recherche-action menée à Nice, en 2007, par l'ERTE I3DL. L'équipe a rassemblé 183 enseignants stagiaires, représentant la plupart des domaines disciplinaires du second degré (professeurs documentalistes, des lycées et collèges, des lycées professionnels et assimilés), et répartis sur deux sites (Nice, Draguignan). Lors des journées de formation (initiale pour les uns, continue pour d'autres), les enseignants stagiaires ont été appelés, en début de session, à produire un récit à l'appui de la question suivante (en 60 à 90 minutes) : "Qu'est-ce qui, dans votre parcours personnel, vous a amené(e) vers le ou les domaine(s) où vous enseignez?» Les feuillets, anonymés - et donc numérotés et brièvement informés -, ont été recueillis par la suite avec la possibilité d'être repris, au terme de la formation, au cas où les stagiaires comptaient y apporter des compléments ou des modifications.

En termes de recherche, l'une des principales questions qui ont été posées au corpus a consisté à déterminer dans quelle mesure de tels supports de formation permettent de construire, au-delà du caractère anecdotique de certains évènements du quotidien et du vécu expérientiel, une identité socio-discursive à travers des regards portés sur les faits et les expériences à la fois personnelles et professionnelles, favorisant ainsi, en quelque sorte, une approche inédite de la professionnalisation, à partir de différents modes d'argumentation et de justification.

\section{Pratiques discursives et rapports aux savoirs d'expérience}

Une première approche des démarches discursives représentées dans les productions nous a amené à distinguer les réponses correspondant à des récits de vie à proprement parler, de celles qui correspondent à d'autres formes de productions, ces dernières ne combinant pas forcément narrativisation et biographisation (voir Pineau, 2006; DeloryMomberger et al., 2007). Ce qu'a révélé le corpus dans un premier temps, c'est que sur 183 feuillets (dont un apparait sans texte), 22 ne correspondent aucunement à des récits de vie, soit $12 \%$ des supports. Parmi ces 22 réponses, les refus à proprement parler catégoriques de répondre (qui sont une forme de réponse en soi) concernent 5 supports, lesquels se présentent soit sous la forme d'une page blanche (120), soit sous celle d'une récusation plus ou moins justifiée de la sollicitation, dont nous reportons ci-après deux exemples ${ }^{5}$ :

5. Nous renvoyons aux numéros des feuillets. 
Pour des raisons personnelles je ne souhaite pas raconter cette partie de ma vie privée (77)

Récit de vie : Je ne souhaite pas m'expliquer sur ce sujet (91).

Hormis la page blanche, laquelle est difficile à interpréter sur le plan du rapport à soi-même et au savoir (notamment en ceci qu'elle révèle peut-être surtout un rapport à la démarche de formation elle-même), il apparait que les refus de produire un récit sur soi sont principalement dus à son caractère intrusif, mais aussi au fait que les participants ont rejeté la possibilité, non pas de produire un discours professionnel, mais d'envisager le récit biographique comme tel.

Dans les productions concernées, la plupart des auteurs concèdent une certaine difficulté à se positionner, tout en formulant plus ou moins concrètement des attentes de formation, comme dans la contribution suivante (16) :

La conscience d'avoir pleinement intégré le statut de professeur n'étant pas acquise, je ne peux avoir le recul nécessaire pour évoquer le «déclic » m'ayant fait passer du statut d'étudiant à celui de professeur.

Parmi les autres contributions, une tendance intermédiaire se dégage, avec des productions dont l'une des particularités est de se présenter de manière plus énumérative, et de s'organiser comme une suite de justifications et/ou d'explications brèves, comme en témoigne la production ci-après (141) :

J'ai toujours aimé les mathématiques, car c'est une science logique, précise.

J'aime transmettre un savoir les mathématiques (je le faisais étant au collège et lycée même après : j'expliquais souvent à mes frères et sœurs les mathématiques).

J'ai toujours aimé les chiffres en plus et le fait que ce soit

Je pense que les autres matières sont chacune vraiment différentes des miennes mais un grand lien les lie : les élèves et la transmission du savoir.

Une telle démarche prend plusieurs formes, à partir desquelles les auteurs questionnent leur parcours individuel plus ou moins à l'écart soit de la biographisation, soit de la narrativisation, soit des deux simultanément, comme celui-ci (5) :

Je ne suis pas très à l'aise avec l'intitulé «Récit de vie». je me bornerai donc à mentionner quelques «idées fortes» : 
- la parole du professeur de français, depuis le Lycée, me semblait disposer d'un pouvoir quasi-magique

- le latin, puis le grec ancien m'ont vite attirée, séduite, passionnée.

- Des études de lettres classiques semblaient donc tout indiquées!

- ... l'aboutissement d'un tel parcours : me voilà !

Les termes qu'emploie l'auteur du feuillet (5) sont de notre point de vue particulièrement significatifs, en ce qu'ils sont plus allocentrés encore que dans la production précédente, où le «je» nous semble toutefois, pour ainsi dire, pseudo-implicatif (comme le serait un je quasiimpersonnel). Les auteurs, dans les deux cas, ne s'y présentent pas en tant que sujets d'un parcours, mais décrivent l'existant comme l'«aboutissement», en quelque sorte hic et nunc, d'une suite d'évènements singuliers et de sensibilités. La construction de l'identité socio-discursive s'établit ainsi dans une faible mesure à la charnière de la production du savoir et de sa transmission. Elle se présente plutôt comme le résultat d'une somme linéaire de rapports aux savoirs académiques ou de représentations liées aux connaissances partagées, voire, éventuellement, à leur dimension épistémologique. Là où une narrativisation plus effective implique, dans presque une moitié des productions (que nous présentons dans la partie «Quand narration et biographie se combinent effectivement»), une déconstruction du rapport à l'expérience (laquelle déconstruction permet, dans le même temps, de lui donner une autre signification), les productions 141 et 5 , comme c'est le cas de près de $45 \%$ des éléments du corpus, reviennent surtout sur la dimension académique du savoir. Elles convoquent l'expérience à travers des pratiques quotidiennes, leur justification sociale, et notamment une énumération de contenus qui amène les auteurs à faire intervenir plusieurs généralités sur la profession. Certaines de ces productions vont d'ailleurs jusqu'à donner une version inventoriée du parcours professionnel, dont voici une illustration (33) :

Éducation musicale

- Notre discipline est particulière. Elle lie une pratique vraiment active à des connaissances théoriques.

- Lors de mes études musicales, je me suis attaché à pratiquer mon instrument. Je me destinais à l'enseignement en école de musique > prof d'instrument.

- Or j'ai eu envie d'enseigner la musique dans sa globalité > pratiquer, connaitre, et percevoir. 
- De plus, ce métier me permet d'envisager l'instrument dans une pratique amateur. Métier le musicien (sic) est trop précaire. Cela me permet de vivre avec ma passion et non d'en vivre.

Ces discours professionnels ne reportent certes pas à des ressources documentaires, ni à une confrontation d'apports de la recherche, mais ils renvoient dans bien des cas à des pratiques de justification qui consistent à conforter l'existant, un rapport au savoir établi, avec ce que cela implique en termes de préconstruction. En souscrivant dans une moindre mesure à la démarche qui consiste à resignifier les éléments d'un parcours, ils sollicitent peu les savoirs expérientiels, mais ils insistent toutefois, avec une certaine productivité, sur le rapport aux savoirs académiques, dont ils commentent certaines caractéristiques en lien plus ou moins direct avec des éléments du vécu.

\section{Quand narration et biographie se combinent effectivement}

Les autres productions, qui ne sont donc ni réfutatives ni commentatives, donnent soit l'intégralité du cursus personnel qui a mené le participant vers l'existant, avec une narration biographique qui cherche à donner une vue d'ensemble d'un parcours individuel, soit un résumé, linéaire ou non, de ce qui en forme les variables principales, en s'appuyant sur certains éléments spécifiques du parcours individuel. Dans les deux cas, il existe effectivement une appropriété de la réponse à la question posée et aux enjeux de la narration sur soi, mais le discours professionnel tenu sur le rapport au savoir et sur l'expérience personnelle n'est pas tout à fait similaire. Pour illustrer notre propos, nous donnerons un exemple de récit qui tend à décrire l'intégralité d'un parcours (86) :

Née dans une famille d'entrepreneurs, j'ai grandi au contact direct avec la culture d'entreprise.

Le choix du parcours d'étude s'est fait «naturellement». Après avoir suivi une année dans une section scientifique, je me suis tout de suite rendu compte que j'avais besoin de l'économie et du droit en tant que «matières vivantes». En effet, j'ai ressenti les disciplines scientifiques comme des disciplines moins liées à la vie quotidienne et trop théoriques.

J'ai donc poursuivi mes études en économie et droit avec une maitrise et un master. J'ai décidé de me spécialiser en finance avec un MBA aux États-Unis. C'est aux États-Unis que j'ai eu ma première expérience de professorat dans une grande école de commerce suite à la réussite d'un examen. 
Peut-être un peu prématurée, j'ai méprisé cette expérience et j'ai eu envie d'une expérience professionnelle importante dans le monde de la finance. C'est ainsi que j'ai travaillé pendant 10 ans en tant qu'analyste financier, partageant mon temps entre Europe et États-Unis.

Au bout de 10 ans de carrière épanouissante mais fatigante, j'ai voulu changer de métier sans changer de matière! J'ai voulu transmettre mes savoirs et mon expérience pour les rendre vivants et j'ai reconsidéré ma première expérience de professorat avec les yeux de la maturité!

Voici, ci-après, un exemple de récit qui, plus sommaire, n'en donne à proprement parler que les grandes lignes, en présentant le parcours par l'intermédiaire d'un fil conducteur réduit à sa plus simple expression (31) :

Après avoir travaillé à mon compte en graphisme (infographie), design produit et architecture intérieure et désireux de faire passer des connaissances et savoir faire - je j'ai pendant 2 ans donné des cours comme contractuel en technologie. Effectivement cette matière correspond le plus à mon vécu et mes savoir faire.

Ces récits spontanés reviennent variablement sur les contextes socioculturels dans lesquels sont apparus les éléments les plus déterminants du parcours personnel, dont ils procurent des représentations variées (nous parlons de récits «spontanés» dans la mesure où il ne s'agit pas d'entretiens biographiques, dont on peut dire qu'ils sont «interactionnels » à la suite de Chanfrault-Duchet [1988], ni de récits dits «de synthèse », tels qu'ils sont exposés par Delory-Momberger [2005] et Snoeckx [2007]). Pour autant, le fait que certains récits soient plus sommaires que d'autres n'a que peu d'influence sur la démarche de biographisation. Comme le note à ce sujet Lindón (2005, p. 59) :

en racontant sa vie, l'individu la repense; d'une façon générale, on peut dire qu'il devient de ce fait le «sociologue de sa propre biographie», car il cherche à établir des connexions entre les faits, il construit ses propres séquences d'évènements, en en sélectionnant certains et en en excluant d'autres.

Dans le récit 86 , l'auteur organise son récit en le soumettant à une linéarité qui lui permet de dégager les moments déterminants de son parcours, tout en établissant une distinction entre plusieurs contextes socio-culturels (celui de l'entreprise, celui de ce qu'il appelle le «professorat»), avec pour principaux éléments significatifs un ensemble de savoirs académiques et de savoirs d'expérience vis-à-vis desquels le 
regard a changé. Comme dans une partie significative des $43 \%$ de récits qui présentent une démarche similaire dans notre corpus, la narrativisation opérée donne lieu à une réorganisation des multiples possibles que suppose un regard vers le passé, à partir de ce qui caractérise, précisément, ce que l'expérience implique en termes de rapport aux savoirs. Dans le cas présent, cette modification se résume dans le passage de la construction du savoir à sa transmission, que l'auteur ne s'est senti en mesure de pratiquer qu'après avoir confronté les savoirs académiques aux savoirs d'expérience. Or, c'est la même démarche qui, quoique esquissée, apparait dans 31 , même si la production biographique est moins évènementialisée, et que la distinction entre plusieurs contextes socio-culturels n'est pas aussi nettement établie.

Le récit biographique permet ici, à leurs auteurs, d'établir des rapports entre les pratiques professionnelles et les représentations qu'ils s'en font dans la perspective d'une formation suivie. À ce titre, il contribue à construire une représentation de soi plurielle que l'on peut rapprocher de la question d'identités professionnelles en construction, ce qui permet de le placer dans un suivi individualisé, et d'apporter des éléments de réponse à un moment où les étudiants, par exemple, sont appelés à se déterminer dans leurs parcours de formation (en particulier en deuxième année de master).

Alors que les récits sur soi peuvent sembler contourner l'exigence qui consiste à réfléchir sur le rapport aux savoirs, en ce qu'ils n'en font pas le principe organisateur, les productions qui souscrivent entièrement à la démarche démontrent en partie le contraire, en ce qu'elles permettent, par l'intermédiaire d'un retour sur soi, de mener un questionnement sur les savoirs plus particulièrement expérientiels.

\section{Quelques conclusions}

La réflexion menée au cours de cette première analyse nous a conduit à établir qu'à partir du moment où le récit de vie spontané est envisagé (et pratiqué) comme un discours professionnel, il correspond soit à une «autonarration» (Schmitt, 2008), soit à une démarche que nous appellerons, à la suite de Gasparini (2008, p. 295), d' «autocommentaire». À cela s'ajoute le refus bien sûr, qui existe bel et bien, mais que l'on peut considérer comme marginal. Ces tendances témoignent dans l'ensemble, au moins en partie, de la diversité des implications et des représentations concernant la profession, mais aussi d'une possible 
complémentarité, en formation, entre les productions valorisant les produits de la recherche et celles qui valorisent les parcours individuels.

Les productions qui répondent au minimum à cette démarche de formation intègrent donc des temporalités diverses, et par là même des rapports différents aux savoirs d'expérience. L'autonarration rend compte d'un parcours dont l'existant, contemporain du récit, est présenté comme une forme d'accomplissement (quand bien même il serait envisagé comme provisoire), et s'inscrit dans une évènementialité qui organise la production biographique et le rapport aux savoirs. L'autocommentaire, de son côté, est en tout ou partie anhistorique et part de l'existant, à proprement parler du rapport aux savoirs, en recourant variablement à des évènements vécus. D'un point de vue argumentatif, il semble que les deux démarches se distinguent d'autant plus ouvertement que la première s'apparente à une reconstruction/resignification, alors que la deuxième se rapproche à bien des égards d'une pratique de justification. Or, il est possible que ces deux approches témoignent de demandes sociales différentes vis-à-vis du métier et de la formation, appelant par là même deux formes distinctes d'accompagnement. Ainsi, alors que les autocommentaires constituent à notre sens un matériau particulièrement productif dans le cadre de groupes de travail ou dans un processus de tutorat (Draperi, 2002, p. 395 et suiv.; Bliez-Sullerot et Mevel, 2004), les autonarrations, en plus de leur capacité à intégrer ces formes d'accompagnement, permettent de poser encore plus clairement la question d'une formation tout au long de la vie.

Dans tous les cas, le recours au récit biographique nous parait présenter une opportunité non négligeable en formation, en ce qu'il constitue un discours professionnel spécifiquement porté sur le rapport à soi-même et aux savoir-être des participants. À partir du moment où ces derniers sont appelés à se positionner dans le cadre de métiers où le rapport à soi (et à l'autre) en rend l'apprentissage quelquefois difficile, une telle approche permet d'accompagner concrètement les personnes et de les sensibiliser sur ce qui forme les singularités de leur parcours.

\section{RÉFÉRENCES BIBLIOGRAPHIQUES}

BertaUX D. (1997) : Les Récits de vie, perspective ethnosociologique, Paris, Nathan sociologie (128).

Blanc N. et Varga R. (dir.) (2006): Lidil, n 34, Rapport de stage et mémoire professionnel. Normes, usages et représentations, Grenoble, Ellug. 
Blanchard-Laville C. et Fablet D. (dir.) (2000) [1998] : Analyser les pratiques professionnelles, Paris, L'Harmattan.

Bliez-Sullerot N. et Mevel Y. (2004) : Récits de vie en formation, l'exemple des enseignants, Paris, L'Harmattan.

Chanfrault-Duchet M.-F. (1988) : «Le système interactionnel du récit de vie», Sociétés, nº 18 , p. 26-31.

CRos F. (dir.) (2006) : Écrire sur sa pratique pour développer des compétences professionnelles, Paris, L'Harmattan.

De Halleux M. (2008) : «Histoires de vie et formation des éducateurs », Pensée Plurielle, $\mathrm{n}^{\circ} 18$ (2), p. 161-177.

Delory-Momberger Chr. (2000) : Les Histoires de vie. De l'invention de soi au projet de formation, Paris, Anthropos.

- (2005) : Histoire de vie et recherche biographique en éducation, Paris, Anthropos.

Delory-Momberger Chr., Boutinet J.-P., Biarnes J., Feldhendler D., Dispagne M., Schaller J.-J. et Mezza J. (2007) : Insertion, biographisation, éducation : champs et problématiques de l'insertion, numéro de L'Orientation scolaire et professionnelle, $\mathrm{n}^{\circ} 36$ (3).

Demazière D. (2008) : «L'Entretien biographique comme interaction. Négociations, contre-interprétations, ajustements de sens », Langage et Société, $\mathrm{n}^{\circ} 123$, p. 15-36.

De Villers G. (1993) : «L'Histoire de vie comme méthode clinique», Cahiers de la section des sciences de l'éducation de l'université de Genève, $\mathrm{n}^{\circ} 72$, p. 135-155.

DrAPERI J.-F. (2002) : «L'Entretien auto-biographique : présentation abrégée et commentaires pratiques», dans A. Moisan et P. Carré (dir.), L'Autoformation, fait social? Aspects historiques et sociologiques, Paris, L'Harmattan, p. 375-392.

Gasparini Ph. (2008) : Autofiction, Paris, Le Seuil.

Guigue M. (2000) : «Écrire en formation d'enseignants - Écrire ses pratiques, entre leurre et formation réflexive», Cahiers Pédagogiques, 55, $\mathrm{n}^{\circ} 388-389$.

Legrand M. (1993) : L'Approche biographique, Paris, Epi.

Lindón A. (2005) : «Récit autobiographique, reconstruction de l'expérience et fabulation : une approximation à l'action sociale», Sociétés, $\mathrm{n}^{\circ} 87$ (1), p. 55-63.

Pineau G. (2002) : Les Histoires de vie, Paris, PUF.

- (2006) : «As histórias de vida em formação: gênese de uma corrente de pesquisa-ação-formação existencial »,Educ. Pesqui., vol. 32, n 2 , p. 329-343.

Riceur P. (1975) : La Métaphore vive, Paris, Le Seuil. 
Schmiтt A. (2008) : «Autonarration et fiction du réel», communication au colloque Autofiction du Centre culturel international de Cerisy (coord. C. Burgelin et I. Grell), juillet 2008.

SNOECKX M. (2007) : «Écriture professionnelle et explicitation : autobiographie professionnelle en formation» [en ligne], Expliciter, $\mathrm{n}^{\circ}$ 70. Disponible sur <http://www.explicitation.ch/documents/AutobiographieMir. pdf $>$ [consulté le 4 avril 2011].

Torterat F. (2008) : «L'entretien d'accompagnement professionnel, entre problématisation et co-désignation », Spirale, n 41, p. 49-61.

- (2009) : «Un bref exemple de réflexion sur corpus oraux en milieu professionnel», dans M.-L. Martinez (éd.), Identités en éducation et en formation à l'école, Paris, L'Harmattan, à paraitre. 